Supporting Information

\title{
Hybrid polydimethylsiloxane bioscaffold-intravascular catheter for cellular therapies
}

Sophia $\mathrm{Hu}^{1 *}$, Rosita Primavera ${ }^{1 *}$, Mehdi Razavi ${ }^{2 *}$, Anirudh Avadhani ${ }^{1}$, Jing Wang ${ }^{1}$, Avnesh S Thakor ${ }^{1 *}$

${ }^{1}$ Interventional Regenerative Medicine and Imaging Laboratory, Department of Radiology, Stanford University, Palo Alto, California, 94304, USA.

${ }^{2}$ Biionix $^{\mathrm{TM}}$ (Bionic Materials, Implants \& Interfaces) Cluster, Department of Internal Medicine, College of Medicine, University of Central Florida, Orlando, Florida 32827, USA

${ }^{*}$ Correspondence: asthakor@stanford.edu, 3155 Porter Drive, Palo Alto, CA 94305

Supporting information 1: Materials and methods, 3 pages

Supporting information 2: CFD parameters, 4 pages, 5 tables, 2 figures

Supporting information 3: Supplementary results, 2 pages 


\section{SUPPORTING INFORMATION 1: MATERIALS AND METHODS}

\section{Computational fluid simulation studies}

3D models of the hybrid PDMS bioscaffold-intravascular catheter were designed using Solidworks (2017) and Autodesk Inventor Professional (2018). Fluid dynamics were evaluated using Autodesk CFD (2017). We simulated both $14 \mathrm{~F}$ and $4 \mathrm{~F}$ size catheters for use in the internal jugular vein. ${ }^{1,2}$ The $14 \mathrm{~F}$ catheter model was constructed with a $4.0 \mathrm{~mm}$ inner lumen diameter and $4.6 \mathrm{~mm}$ outer lumen diameter and the blood vessel was represented as an extruded cylinder of $20 \mathrm{~mm}$ surrounding the catheter. The $4 \mathrm{~F}$ model was constructed with a $1.2 \mathrm{~mm}$ inner lumen diameter and $1.5 \mathrm{~mm}$ outer lumen diameter and the blood vessel was simulated as an extruded cylinder of $20 \mathrm{~mm}$ surrounding the catheter. The catheter and blood vessel were assigned the materials of silicon rubber and blood, respectively, in Autodesk CFD. The bioscaffold was simulated as an internal cylinder tangent to the lumen face of the catheter; the porous nature of the bioscaffold was modeled as a resistance to fluid flow with a flow coefficient $=0.8$. Boundary conditions included: $20 \mathrm{~cm} / \mathrm{s}$ flow velocity, oxygen diffusion coefficient $=1.6 \mathrm{e}-5$ (using a general scalar assigning the blood and bioscaffold of relative values 1 and 0 , respectively), and exit pressure $=0 \mathrm{mmHg}$ (simulating low venous pressure). ${ }^{1}$ A full table of the CFD parameters can be found in Supporting Information 2. We solved the system under non-Newtonian blood flow conditions for water age and oxygen diffusion across the length of the catheter to determine the best model. Simulations were run for 100 iterations with approximately 120,000 mesh elements per model.

\section{Catheter materials}

Medical-grade polyurethane catheters (Nordson MEDICAL, New Hampshire, USA) were used for all experiments. To provide a support matrix for islets, PDMS bioscaffolds were synthesized within the catheter via solvent casting and particulate leaching technique. First, a silicone elastomer base (RTV 615A) was mixed with the curing agent (RTV615B) in a 4:1 ratio (Momentive Performance Materials). This was then mixed with sodium chloride (150 $\mu \mathrm{m}-250 \mu \mathrm{m}$ particles, Fisher Chemical) to form 30, 50, 60, 70, and $80 \%$ bioscaffold porosities. The PDMS-salt mixture was pushed into the catheter and placed into an oven (Thermo Scientific, Heratherm Oven) at $90^{\circ} \mathrm{C}$ for 7 days to cure the PDMS-salt mixture. After curing, side holes were punctured into the catheter using a soldering tip, and then the catheter was placed into a water shaker (Thermo Scientific, MaxQ 5000) at $200 \mathrm{rpm}$ for 7 days at $37^{\circ} \mathrm{C}$ to dissolve the salt particles and form a porous bioscaffold. Water was exchanged every day to ensure maximum salt dissolution. Bioscaffold porosity and homogeneity were evaluated through scanning electron microscopy (SEM) images (Stanford Nano Shared Facilities). Pore size analysis was manually conducted using the ImageJ software measuring function to avoid regions deformed when obtaining sections. 20 pore measurements were taken across the major axis of the pores per porosity $(n=3)$ to determine average \pm SE pore sizes.

\section{Islet isolation and culture}

All pancreatic islet isolation was conducted in accordance with the Institutional Animal Care and Use Committee (IACUC) at Stanford University. Pancreatic islets were isolated from healthy male mice (C57BL/6 mice from Charles River Laboratories, USA, at 6-8 weeks of age with a weight of 22-25g) using collagenase digestion and Histopaque ${ }^{\circledR}$ gradients solutions $\left(1.119 \mathrm{~g} / \mathrm{mL}\right.$ and $1.077 \mathrm{~g} / \mathrm{mL}$ for Histopaque ${ }^{{ }_{-}}$ 1119 and Histopaque ${ }^{\circledR}-1077$, respectively), as previously described..$^{2}$ In brief, mice were first anesthetized with isoflurane (3-4\% for inhalation) and then euthanized by cervical dislocation. The mouse pancreas was surgically exposed and the pancreatic duct isolated and cannulated. The pancreas was distended using an infusion of 2-3 mL of Hank's balanced salt solution (HBSS, Sigma-Aldrich) supplemented with $0.1 \%$ bovine 
serum albumin (BSA; Sigma-Aldrich) containing $1 \mathrm{mg} / \mathrm{mL}$ of collagenase VI (Sigma-Aldrich). The pancreas was then dissected and incubated for $10 \mathrm{~min}$ in a water bath at $37^{\circ} \mathrm{C}$. Islets were purified by gradient centrifugation on Histopaque ${ }^{\circledR}-1119$ and Histopaque ${ }^{\circledR}-1077$ (Sigma-Aldrich), and then individually handpicked and cultured in culture petri dishes containing RPMI 1640 medium without Phenol Red supplemented with $10 \%$ fetal bovine serum (FBS).

Human islets were provided by the Alberta Diabetes Institute IsletCore at the University of Alberta in Edmonton with the assistance of the Human Organ Procurement and Exchange (HOPE) program, Trillium Gift of Life Network (TGLN) and other Canadian organ procurement organizations. Islet isolation was approved by the Human Research Ethics Board at the University of Alberta (Pro00013094). All donors' families gave informed consent for the use of pancreatic tissue in research. Islets were processed as previously described by Lyons et al. ${ }^{3}$ In brief, islets were isolated within 24 hours of donor death and cultured in CMRL 1066 medium. They were distributed the next business day and cultured within 6 hours of receipt in RPMI 1640 medium supplemented with 10\% fetal bovine serum.

\section{Cell seeding}

Mouse islets were seeded into the hybrid PDMS bioscaffold-intravascular catheters via pipetting. Specifically, islets were viewed under a stereomicroscope, hand-picked with a $50 \mu \mathrm{L}$ pipette to ensure an even distribution of sizes, and then seeded into open-ended sections of the catheter only (without PDMS), PDMS bioscaffold only (without the catheter), or the catheter containing the PDMS bioscaffold. To seed the catheter prototypes with human islets, islets were collected and centrifuged in RPMI medium (RPMI 1640, supplemented with $10 \%$ FBS) at $1200 \mathrm{RPM}$ for $3 \mathrm{~min}$. After removing the supernatant, cells were resuspended in $10 \mathrm{~mL}$ RPMI (RPMI 1640, supplemented with 10\% FBS) and picked with a $50 \mu \mathrm{L}$ pipette under a light microscope. Islets were then directly seeded into the catheter prototype.

\section{Liveldead assay}

Mouse islets in all conditions were incubated for 24 hours in a 96-well plate (20 islets/well). Islet viability was measured with fluorescence microscopy using a live/dead assay. Islets alone served as the control group. After 24 hours incubation, both control and experimental groups were stained with a solution of $8 \mu \mathrm{L}$ of fluorescein diacetate (FDA, $5 \mathrm{mg} / \mathrm{mL}$ ) $+50 \mu \mathrm{L}$ of propidium iodide (PI, $2 \mathrm{mg} / \mathrm{mL}$ ) in a 96well plate and incubated for 5 minutes. They were then imaged using fluorescence microscopy (Leica Microscopy, California, USA) to assess islet viability. Live/dead analysis was conducted using Image J colorimetric quantification.

Human islet viability was evaluated using the Live/Dead fluorescence staining solution as previously described by Karaoz et al. to test islet biocompatibility with the catheter. ${ }^{4}$ Prior to their shipment from the University of Alberta, islet viability was evaluated using fluorescence-activated cell sorting. After incubation with Live/Dead staining solution, the samples were imaged using fluorescence microscopy (Leica Microscopy, California, USA) at 20x magnification. They were categorized into groups of $0 \%, 25 \%$, $50 \%, 75 \%$ and $100 \%$ viability using the widefield fluorescence images and the results were summed together to obtain an average islet survival value. The islet viability assay was conducted on three separate samples with 30 islets in each sample from the control, catheter, PDMS bioscaffold, and hybrid catheter with PDMS bioscaffold groups. 


\section{Glucose stimulated insulin sensitivity assay}

The functionality of islets seeded within hybrid PDMS bioscaffold-intravascular catheters were tested by examining their insulin secretion profiles using a glucose stimulated insulin secretion (GSIS) assay. A 12-well plate containing Transwell ${ }^{\circledR}$ with $0.4 \mu \mathrm{m}$ pore polyester membrane inserts (Corning ${ }^{\circledR}$, USA) was used to prevent islets from being inadvertently aspirated when changing glucose solutions $(n=5)$. The Transwell ${ }^{\circledR}$ inserts were primed with $100 \mu \mathrm{L}$ cell culture medium (RPMI without Phenol Red, 10\% FBS) 30 minutes prior to beginning the GSIS assay. After isolation, islets were incubated overnight and then the specified number of islets (20 islets) were hand-picked up under stereomicroscopy using a $50 \mu \mathrm{L}$ pipettor. Islets were incubated in $1 \mathrm{~cm}$ sections of the catheter only (without PDMS), PDMS bioscaffold only (without the catheter), and the catheter containing the PDMS bioscaffold. Each section was placed into the top well of the Transwell ${ }^{\circledR}$ insert. For the control group, islets were handpicked and placed directly into the top wells of the Transwell ${ }^{\circledR}$ insert achieving 20 islets in $200 \mu \mathrm{L}$ of complete medium (RPMI without Phenol Red, $10 \%$ fetal bovine serum (FBS; Invitrogen, USA)). Both the control and the experimental groups were incubated with $200 \mu \mathrm{L}$ of low glucose $(2.3 \mathrm{mM}$ glucose in Krebs Ringer Bicarbonate Buffer $(137 \mathrm{mM} \mathrm{NaCl}$, $4.7 \mathrm{mM} \mathrm{KCl}, 1.2 \mathrm{mM} \mathrm{KH}_{2} \mathrm{PO}_{4}, 1.2 \mathrm{mM} \mathrm{MgSO}_{4}-7 \mathrm{H}_{2} \mathrm{O}, 2.5 \mathrm{mM} \mathrm{CaCl}_{2}-2 \mathrm{H}_{2} \mathrm{O}, 25 \mathrm{mM} \mathrm{NaHCO}$ ), LG-1) in the bottom well of the Transwell ${ }^{\circledR}$ system at $37^{\circ} \mathrm{C}$ and $5 \% \mathrm{CO}_{2}$. After 1 hour, the supernatant in the bottom compartment was collected and immediately stored in $-80^{\circ} \mathrm{C}$. This process was repeated with high glucose (20 mM glucose in Krebs Ringer Bicarbonate Buffer, HG) and low glucose (2.3 mM glucose in Krebs Ringer Bicarbonate Buffer, LG-2). The supernatant was analyzed using the Mercodia Mouse Insulin ELISA kit.

\section{Statistical analysis}

All in vitro experiments were performed in $\mathrm{n}=5$, and the results expressed as the mean \pm standard error of the mean. The statistical analysis was performed using a Student's t-test or one-way ANOVA with post-hoc Tuckey test. Differences were considered statistically significant with $p<0.05$. 


\section{References}

1) Goldstick, T. K.; Ciuryla, V. T.; Zuckerman, L. Diffusion of Oxygen in Plasma and Blood. Adv. Exp. Med. Biol. 1976, 75, 183-190.

2) Ren, G.; Rezaee, M.; Razavi, M.; Taysir, A.; Wang, J.; Thakor, A. S. Adipose Tissue-Derived Mesenchymal Stem Cells Rescue the Function of Islets Transplanted in Sub-Therapeutic Numbers via Their Angiogenic Properties. Cell Tissue Res. 2019, 376, 3, 353-364.

3) Lyon, J.; Manning Fox, J. E.; Spigelman, A. F.; Kim, R.; Smith, N.; O'Gorman, D.; Kin, T.; Shapiro, A. M. J.; Rajotte, R. V.; MacDonald, P. E. Research-Focused Isolation of Human Islets From Donors With and Without Diabetes at the Alberta Diabetes Institute IsletCore. Endocrinology 2016, 157, 560-569.

4) Karaoz, E.; Genç, Z. S.; Demircan, P. Ç.; Aksoy, A.; Duruksu, G. Protection of Rat Pancreatic Islet Function and Viability by Coculture with Rat Bone Marrow-Derived Mesenchymal Stem Cells. Cell Death Dis. 2010, 1, e36-e36. 
SUPPORTING INFORMATION 2: CFD Parameters

\section{Table S1: Materials}

\begin{tabular}{|l|ll|}
\hline Material & Properties & \\
\hline Blood & Density & $1003.0 \mathrm{~kg} / \mathrm{m} 3$ \\
& Viscosity & NonNewt Pwr Law \\
& Conductivity & $0.6 \mathrm{~W} / \mathrm{m}-\mathrm{K}$ \\
& Specific heat & $4182.0 \mathrm{~J} / \mathrm{kg}-\mathrm{K}$ \\
& Compressibility & $2185650000.0 \mathrm{~Pa}$ \\
& Emissivity & 0.7 \\
& Wall roughness & 0.0 meter \\
& Phase & Vapor Pressure \\
\hline Silicon Rubber & X-Direction & $0.7 \mathrm{~W} / \mathrm{m}-\mathrm{K}$ \\
& Y-Direction & Same as X-dir. \\
& Z-Direction & Same as X-dir. \\
& Density & $1.7 \mathrm{~g} / \mathrm{cm} 3$ \\
& Specific heat & $0.7 \mathrm{~J} / \mathrm{g}-\mathrm{K}$ \\
& Emissivity & 0.9 \\
& Transmissivity & 0.0 \\
& Electrical resistivity & 0.0 ohm-cm \\
& Wall roughness & $0.0 \mathrm{~meter}$ \\
\hline Default_Resistance & Through-Flow K & Free Area Ratio \\
& Normal direction $1 \mathrm{~K}$ & 0.0 \\
& Normal direction $2 \mathrm{~K}$ & 0.0 \\
& Conductivity & $1.0 \mathrm{~W} / \mathrm{m}-\mathrm{K}$ \\
\hline
\end{tabular}




\section{Table S2: Boundary conditions}

\begin{tabular}{|l|l|}
\hline Type & Assigned to \\
\hline Velocity Normal $(20 \mathrm{~cm} / \mathrm{s})$ & $\begin{array}{l}\text { Blood vessel inlet. Models the velocity of blood in the internal jugular } \\
\text { vein. }\end{array}$ \\
\hline Scalar (1) & $\begin{array}{l}\text { Blood outside the catheter. Models the initial oxygenation of blood } \\
\text { outside the catheter. }\end{array}$ \\
\hline Pressure(0 dyne $/ \mathrm{cm} 2$ Gage $)$ & $\begin{array}{l}\text { Blood vessel inlet. Models the exit pressure of the blood vessel, letting } \\
\text { blood exit the vessel. }\end{array}$ \\
\hline Velocity Normal $(0 \mathrm{~cm} / \mathrm{s})$ & Blood vessel outlet. Allows blood to exit the vessel. \\
\hline Scalar(0) & $\begin{array}{l}\text { Blood inside the catheter. Models the initial unoxygenated state of } \\
\text { blood inside the catheter. }\end{array}$ \\
\hline Free Area Ratio (.8) & $\begin{array}{l}\text { Resistance inside the catheter. Models the porosity of the bioscaffold } \\
\text { inside the catheter. }\end{array}$ \\
\hline
\end{tabular}

\section{Table S3: Physics and solver settings}

\begin{tabular}{|l|l|}
\hline Compressibility & Incompressible \\
\hline Heat Transfer & Off \\
\hline Radiation & Off \\
\hline Scalar & General scalar \\
\hline Turbulence & On \\
\hline Solution mode & Steady State \\
\hline Intelligent solution control & On \\
\hline Advection scheme & ADV 1 \\
\hline Turbulence model & k-epsilon \\
\hline Iterations run & 100 \\
\hline
\end{tabular}


Table S4: Mesh settings (14F)

Automatic Meshing Settings

\begin{tabular}{|l|l|}
\hline Surface refinement & 0 \\
\hline Gap refinement & 0 \\
\hline Resolution factor & 0.8 \\
\hline Edge growth rate & 1.1 \\
\hline Minimum points on edge & 2 \\
\hline Points on longest edge & 10 \\
\hline Surface limiting aspect ratio & 20 \\
\hline
\end{tabular}

Mesh Enhancement Settings

\begin{tabular}{|l|l|}
\hline Mesh enhancement & 1 \\
\hline Enhancement blending & 0 \\
\hline Number of layers & 3 \\
\hline Layer factor & 0.45 \\
\hline Layer gradation & 1.05 \\
\hline
\end{tabular}

Figure S1: Meshed Model

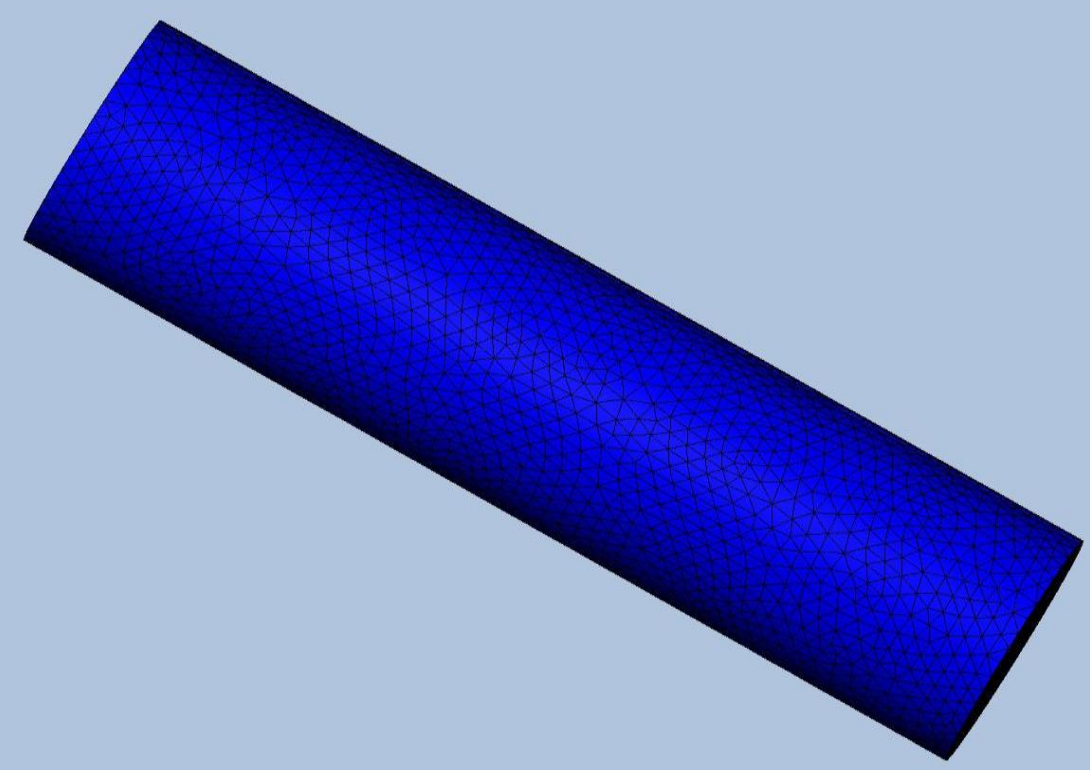


Table S5: Mesh settings (4F)

Automatic Meshing Settings

\begin{tabular}{|l|l|}
\hline Surface refinement & 0 \\
\hline Gap refinement & 0 \\
\hline Resolution factor & 0.7 \\
\hline Edge growth rate & 1.1 \\
\hline Minimum points on edge & 2 \\
\hline Points on longest edge & 10 \\
\hline Surface limiting aspect ratio & 20 \\
\hline
\end{tabular}

Mesh Enhancement Settings

\begin{tabular}{|l|l|}
\hline Mesh enhancement & 1 \\
\hline Enhancement blending & 0 \\
\hline Number of layers & 3 \\
\hline Layer factor & 0.45 \\
\hline Layer gradation & 1.05 \\
\hline
\end{tabular}

Figure S2: Meshed Model

Number of Nodes

Number of Elements 


\section{SUPPORTING INFORMATION 3: SUPPLEMENTARY RESULTS}

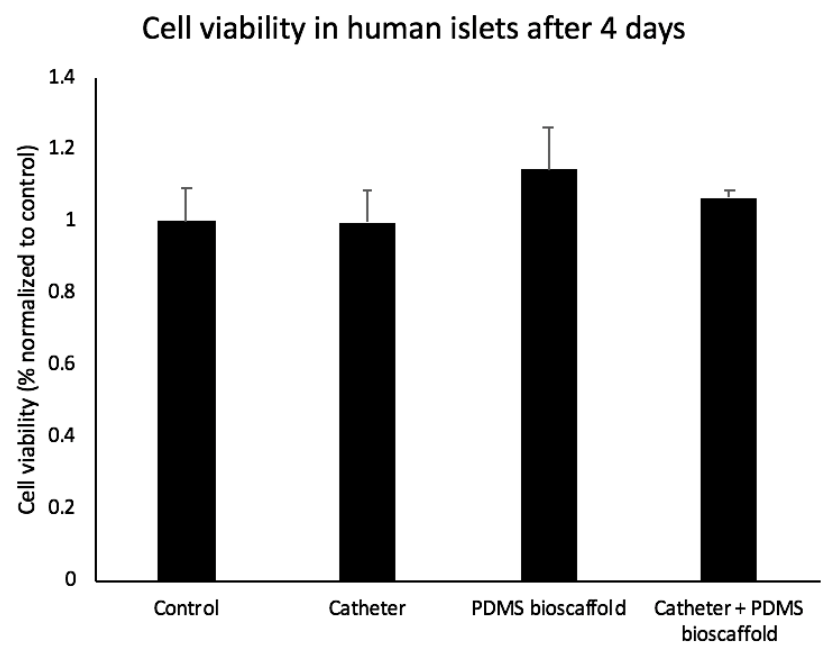

Figure S3. Cell viability of human pancreatic islets following 4 days of culture. Viability was normalized to the control group. Statistical analysis using Student's t-test revealed no significant difference in viability between any of the groups.
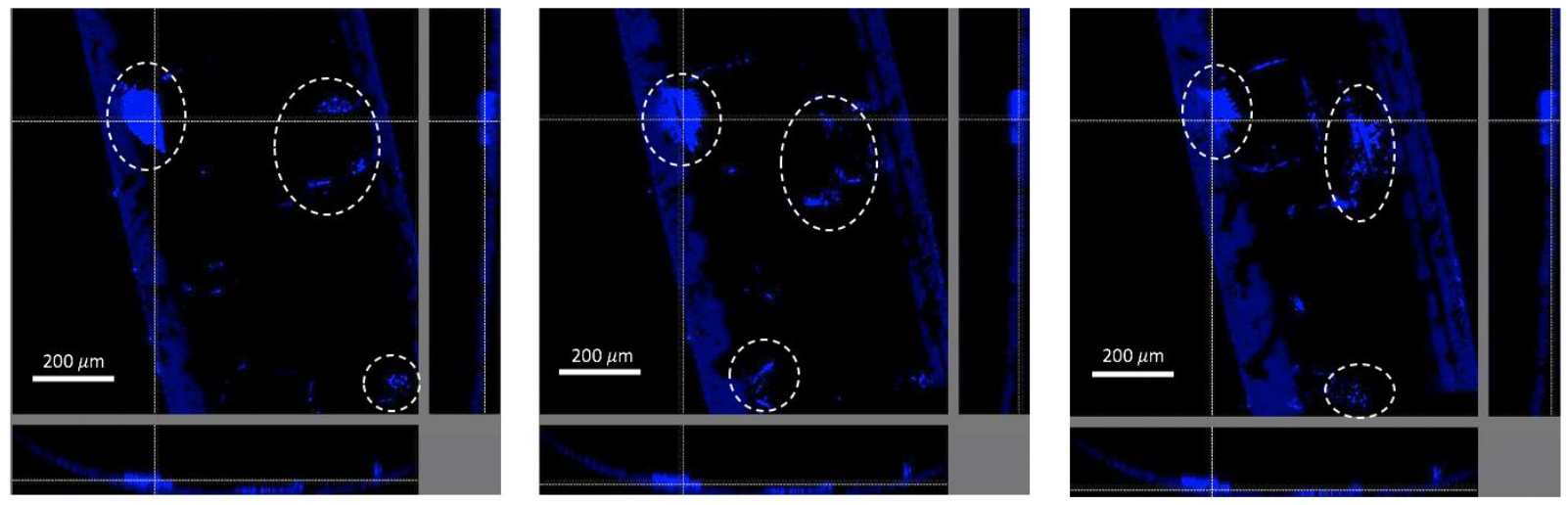

Figure S4. Confocal microscopy analysis (z-stack images) with lateral projections of islets within the hybrid PDMS bioscaffold-intravascular catheter. Islets were stained with Hoechst stain and white dotted circles indicate islets loaded in the scaffold within the catheter." 

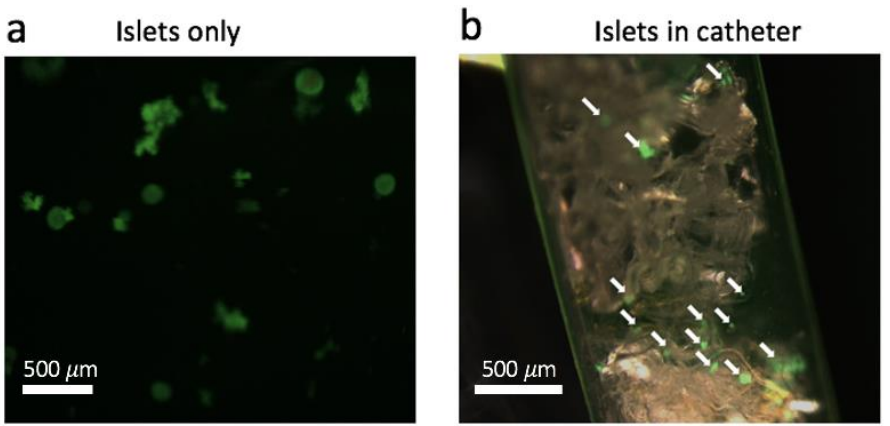

Figure S5. Fluorescence microscopy of the islets only (a) and the islets within the hybrid PDMS bioscaffold-intravascular catheter (b). White arrows indicate islets loaded in the bioscaffold in the catheter. Green (FDA staining) and red (PI staining) fluorescence indicate live and dead cells, respectively. 